\title{
Guidelines for medical audit: seven principles
}

\author{
Charles D Shaw, David W Costain
}

\begin{abstract}
The government, general managers, and professional bodies all agree that medical audit should be implemented throughout the United Kingdom. Nevertheless, it is not yet decided either nationally or locally how audit should be defined and what its implications will be. In an analysis to find ways of measuring the design and effectiveness of hospital audit, therefore, seven main measures emerged that might serve as practical criteria. These were the definition of medical and managerial responsibilities; medical organisation; scope of audit; essential characteristics; resources needed; record keeping; and evaluation.

Though generally consistent with the proposals of the government and the Department of Health, these seven principles offer some alternative approaches.
\end{abstract}

\section{Introduction}

The medical royal colleges and faculties increasingly require evidence of audit before accrediting posts for specialist training, and many have begun to establish other activities such as fellowships, working parties, and seminars to promote the use of audit. At the same time health service managers have identified the need for reviewing clinical work in any comprehensive programme for quality assurance. The government has now explicitly stated that all hospital doctors are expected to participate in audit by 1991 and has detailed its expectations of regional and district health authorities and of clinicians. 'This paper offers criteria by which clinicians and managers in hospital practice may judge whether the service is being audited adequately.

\section{Definition and purpose of audit}

Medical audit is a systematic approach to peer review of medical care in order to identify opportunities for improvement and provide a mechanism for realising them. Medical audit and clinical audit are often used interchangeably, but clinical audit might be considered to cover all aspects of clinical care-for example, nursing and the role of paramedical staff-whereas medical audit relates to practices initiated directly by doctors. It complements and may partly overlap financial audit, utilisation review, and management of resources, but is primarily clinical, not managerial; its focus is the process and results of medical care rather than the use of resources and it is the responsibility of doctors rather than managers.

Medical audit is more systematic, quantified, and formal than traditional clinical ward rounds, meetings, and case presentations but shares with these the objectives of better patient care and education.

Development, London NW1 7NF

Charles D Shaw, MB, director

of medical audit programme

David W Costain, BM,

director of acute service

programme

Correspondence to: $\mathrm{Dr}$ Shaw.

Br Med F 1989;299:498-9
(1) Health authorities and medical staff should define explictly their respective responsibilities for the quality of patient care

Health authorities and managers would not claim to be competent to make judgments on the technical quality of medical care. They must therefore entrust this function to medical staff and have an agreed level of feedback and an assurance that audit exists and is effective in improving patient care. Medical staff should accept corporate responsibility for the quality of medical care both within their own specialty and within the hospital.

A mechanism should be available to tackle issues for which it is uncertain whether responsibility lies with medical staff or with managers.

(2) Medical staff should organise themselves in order to fulfil responsibilities for audit and for taking action to improve clinical performance

At regional health authority level it may be appropriate to have an advisory committee able to give advice, coordinate district activity, and establish mechanisms for audit of regional specialties and district subspecialties. Medical audit throughout the hospital or district should be coordinated by a designated consultant as a recognised clinical commitment with sessions. Support would be provided by a steering group (or audit committee) responsible to the medical staff. The purpose would be to develop a consistent and comprehensive pattern of audit.

Specialty audit should be the responsibility of individual departments or divisions, which would report regularly to the medical staff. Monitoring certain hospital wide policies would remain the responsibility of separate committees (see (7)).

(3) Each hospital and specialty should agree a regular programme of audit in which doctors in all grades participate

Each programme of audit should define priorities for reviewing inpatients, day patients, and outpatients every year. It should recognise that different specialties and case mix require different approaches; in most instances that systematic sampling is more practical than review of every case; and that well tried audit techniques are not available for all aspects of medical care.

Audit should include the use of diagnostic and therapeutic procedures, management of selected clinical conditions, and, when possible, measures of outcome. When clinical responsibility is shared among specialties - for example, admissions from the accident unit, intensive care, and perioperative events-joint audit meetings may be needed. These would include non-medical clinical staff, but all doctors should participate in the audit of their own work and that of their colleagues. Small specialties may need to collaborate on a regional or even supraregional basis in order to provide valid comparisons.

(4) The process of audit should be relevant, objective, quantified, repeatable, and able to effect appopriate change in organisation of the service and clinical practice

The performance of audit and the specific subjects chosen should be relevant to the care of patients and to the training and education of doctors. Priorities might include conditions or treatments characterised by high volume, high risk, high cost, or in which there is particular concern or disagreement over clinical 
management. Individual cases should be selected either at random or systematically by previously agreed criteria.

The definition of explicit criteria or standards is an essential part of the process. Criteria should reconcile local practice with protocols which have already been defined, evaluated, and published. They should be capable of being adapted in response to audit and the development of medical knowledge.

General patterns of practice rather than individual or anecdotal cases should be examined so that conclusions can be reached. Current practice and results should, if possible, be quantified so that they can be compared with other results and over time.

Recommendations for action by management, change in clinical policy, or postgraduate education should be stated explicitly and responsibility for their implementation identified.

\section{(5) Clinicians should be provided with the resources for medical audit}

Audit should be recognised as a legitimate part of the work of clinicians. At least half a session a week should be assigned to educational activities, including medical audit; this will vary among specialties and according to the clerical and technical support available. In addition to regular audit meetings, time will be needed for supervision of data collection, preparation for meetings, documentation, and following up conclusions, as well as for helping other medical staff in developing audit. Allocation of sessional time should be separate from commitments to planning, management, and budgetary control.

Accurate, up to date patient based data should be available in each specialty. Clinicians should personally oversee checking the accuracy of clinical input to hospital data systems. Raw materials include diagnosis, operations, and mortality listings as well as national data for comparisons.

Clerical and technical support should be made available to help clinicians with medical audit. This includes skills in presenting and interpreting data as well as access to relevant material in medical libraries.

\section{(6) The process and outcome of medical audit should be documented}

Data analysis and audit of individual patients and doctors must be confidential. Nevertheless, records should be kept of the data of audit meetings and attendance, issues evaluated, items for improvement identified, actions taken or planned, and results of this action. Regular reports should be made to medical staff and the health authority at an agreed level of detail and frequency so that effective audit is confirmed.

\section{(7) Medical audit should be subject to evaluation}

Doctors, managers, and health authorities should have enough information to evaluate the following items, each of which requires "yes" or "no."

\section{Structure}

1.1. There is formal agreement among health authority, management, and consultant medical staff on responsibility for medical audit.

1.2. Medical staff corporately accept responsibility for the quality of medical care within the hospital.

1.3. There is a named consultant or group, or both, to coordinate medical audit within the hospital.

1.4. Every doctor is assigned to a formal specialty division of the medical staff.

1.5. There are formally constituted groups, including clinical consultants, with responsibility for drugs and therapeutic policy, infection control, postgraduate medical education, ethics, and medical records.

1.6. Time for audit is identified in individual consultant programmes.

1.7. Timely, accurate local data are available to each specialty including diagnostic index, operations and procedure index, and mortality listing.

1.8. Clerical and technical support are allocated for medical audit.

\section{Process}

2.1. Each specialty meets formally and regularly to review clinical work.

2.2. This review:

(a) is attended by all members of the specialty

(b) includes the work of each consultant firm

(c) compares numerically patterns of practice.

2.3. Recommendations are:

(a) recorded for reference

(b) distributed to relevant staff

(c) reviewed to assess progress

2.4. Medical staff receive regular audit reports from each specialty.

2.5. Health authority and management receive a regular summary of process and outcome of audit from medical staff.

\section{Outcome}

3.1. Recommendations lead to documented changes in:

(a) availability of services

(b) organisation of services

(c) written clinical policy

(d) clinical practice.

3.2. Improvement is measured in key issues such as:

(a) complication rates

(b) "avoidable" mortality.

\section{Conclusions}

These guidelines are broadly consistent with those in Working for Patients ${ }^{1}$ and the related working paper on medical audit, ${ }^{2}$ but there are some important differences. Firstly, medical audit is separated from management of resources; the government's inclusion of "the use of resources" in the definition of medical audit is confusing. Secondly, we offer alternative mechanisms for coordination; additional committees dedicated solely to audit may not be necessary. Thirdly, accountability of such committees to the corporate medical staff is clarified, particularly in relation to medical education. Fourthly, behavioural issues may be more challenging than the establishment of technical procedures. Fifthly, comprehensive audit will be limited by time, by resources, and by the availability of audit techniques. And, lastly, the specification of hospital information systems must include data designed for medical audit; these are unlikely to be universally available by the target date of April 1991.

We are grateful to the many individuals and groups of doctors and managers who provided advice and comments in the preparation of this paper.

1 Secretaries of State for Health, Wales, Northern Ireland, and Scotland. Working
for patients. London: HMSO, 1989. (Cmnd 555.)

Department of Health. Working for patients. Medical audit. London: HMSO, 1989. (Working paper No 6.

(Accepted 7 fuly 1989 